\title{
The Integrability and Existence of Periodic Solutions on a First-Order Nonlinear Differential Equation with a Polynomial Nonlinear Term
}

\author{
Ni Hua and Tian Li-Xin \\ Faculty of Science, Jiangsu University, Jiangsu, Zhenjiang 212013, China \\ Correspondence should be addressed to Ni Hua, nihua979@126.com \\ Received 18 July 2011; Revised 19 October 2011; Accepted 23 October 2011 \\ Academic Editor: Oded Gottlieb \\ Copyright (c) 2012 N. Hua and T. Li-Xin. This is an open access article distributed under the \\ Creative Commons Attribution License, which permits unrestricted use, distribution, and \\ reproduction in any medium, provided the original work is properly cited. \\ This paper deals with a first-order differential equation with a polynomial nonlinear term. The \\ integrability and existence of periodic solutions of the equation are obtained, and the stability of \\ periodic solutions of the equation is derived.
}

\section{Introduction}

Consider the following first-order nonlinear differential equation:

$$
\frac{d x}{d t}=\sum_{k=0}^{n} a_{k}(t) x^{n-k} \quad(n \in N, n \geq 2)
$$

when $n=2$, (1.1) becomes Ricatti's equation, when $n=3$, (1.1) becomes the following nonlinear Abel type differential equation:

$$
\frac{d x}{d t}=a(t) x^{3}+b(t) x^{2}+c(t) x+d(t)
$$

The nonlinear Abel type differential equation plays an important role in many physical and technical applications [1-9]. The mathematical properties of (1.2) have been intensively investigated in the mathematical and physical literature. Matsuno [10] analyzed a twodimensional dynamical system associated with Abel nonlinear equation. Strobel and 
Reid [11], Reid and Strobel [12] have obtained superposition rules (prescriptions for combining a finite number of known particular solutions in such a way to obtain the general solution to a (system of) differential equation(s) without operation of integration) for the Abel type equation, involving four or two particular solutions. Mak et al. [13], Mak and Harko [14] have presented a solution-generating technique for Abel type ordinary differential equation, both suppose that $y=y_{1}(x)$ is a particular solution of (1.2), by means of the transformations methods, and present an alternative method of generating the general solution of (1.2) from a particular one.

Zheltukhin and Trzetrzelewski [15] developed the geometric approach to study the dynamics of $\mathrm{U}(1)$-invariant membranes. The approach reveals an important role of the Abel nonlinear differential equation of the first type with variable coefficients depending on time and one of the membrane extendedness parameters. The general solution of the Abel equation was constructed.

However, little work was done about the integrability and periodicity of (1.1). In this paper, we discuss the integrability and the periodic solutions of (1.1); the sufficient conditions which guarantee the integrability and the existence of the periodic solutions for (1.1) are obtained, and the stability of the periodic solutions of (1.1) is discussed. To the best of authors' knowledge, this is the first paper considering the three periodic solutions of (1.1), some new results are obtained.

The present paper is organized as follows. In Section 2, we give three lemmas to be used later. In Section 3, the integrability of (1.1) is derived. In Section 4, the existence and stability of the periodic solutions of (1.1) are obtained. In Section 5, we conclude our results.

\section{Preliminary Lemmas}

For the sake of convenience, suppose that $f$ is a continuous $\omega$-periodic function defined on $R$, we denote $f_{M}=\sup _{t \in[0, \omega]} f(t)$ and $f_{L}=\inf _{t \in[0, \omega]} f(t) ; m(f(t))=(1 / \omega) \int_{0}^{\omega} f(t) d t$.

Consider the following:

$$
\frac{d x}{d t}=x\left[a(t)+b(t) x^{n-1}\right]
$$

$n \in N, n \geq 2, a(t), b(t)$ are continuous functions defined on $R$.

Lemma 2.1. The domain $R_{+}=\{x \mid x>0\}$ is positive invariant with respect to (2.1).

Proof. By (2.1), it follows that

$$
x(t)=x\left(t_{0}\right) \exp \left\{\int_{t_{0}}^{t}\left[a(s)+b(s) x^{n-1}(s)\right] d s\right\}
$$

the assertion is valid for all $x\left(t_{0}\right)>0, t \geq t_{0}$. The proof is completed.

Lemma 2.2. The domain $R_{-}=\{x \mid x<0\}$ is negative invariant with respect to (2.1). 
Lemma 2.3. Consider the following:

$$
\frac{d x}{d t}=a(t) x+b(t)
$$

$a(t), b(t)$ are continuous $\omega$-periodic functions, if $m(a(t)) \neq 0$, then (2.3) exists a unique $\omega$-periodic solution $\eta(t)$, and $\eta(t)$ can be written as follows:

$$
\eta(t)=\frac{\int_{t}^{t+\omega} b(s) e^{\int_{s-\omega}^{t} a(\tau) d \tau} d s}{1-e^{\int_{0}^{\omega} a(\tau) d \tau}}, \quad m(a(t)) \neq 0
$$

or

$$
\eta(t)= \begin{cases}\int_{-\infty}^{t} e^{\int_{s}^{t} a(\tau) d \tau} b(s) d s, & m(a(t))<0, \\ -\int_{t}^{+\infty} e^{\int_{s}^{t} a(\tau) d \tau} b(s) d s, & m(a(t))>0 .\end{cases}
$$

Proof. The proof of Lemma 2.3 is a few, such as that of the papers $[16,17]$ and others, But in [16], the author only proved the case of the almost periodic equation; in [17], the authors just proved that Lotka-volterra equation has a unique globally attractive periodic solution, but they did not give the very two expressions of the periodic solution as above. In order to make the following proof clear, here we give our proof.

From (2.3), it is easy for us to get the unique solution $x(t)$ with the initial value $x\left(t_{0}\right)=$ $x_{0}$ which can be written as follows:

$$
x(t)=e^{\int_{t_{0}}^{t} a(\tau) d \tau} x_{0}+\int_{t_{0}}^{t} b(s) e^{\int_{s}^{t} a(\tau) d \tau} d s,
$$

thus we have

$$
x(t+\omega)=e^{f_{t_{0}}^{t+\omega} a(\tau) d \tau} x_{0}+\int_{t_{0}}^{t+\omega} b(s) e^{\int_{s}^{t+\omega} a(\tau) d \tau} d s
$$

Let $t=t_{0}$, by (2.6) and (2.7), if $x(t)$ is an $\omega$-periodic function, then it follows that $x\left(t_{0}\right)=$ $x\left(t_{0}+\omega\right)$, thus we can get

$$
x_{0}=\frac{\int_{t_{0}}^{t_{0}+\omega} b(s) e^{\int_{s}^{t_{0}+\omega} a(\tau) d \tau} d s}{1-e^{\int_{0}^{\omega} a(\tau) d \tau}} .
$$


4

Mathematical Problems in Engineering

Substitute (2.8) into (2.6), it follows that

$$
\begin{aligned}
x(t) & =e^{\int_{t_{0}}^{t} a(\tau) d \tau} \frac{\int_{t_{0}}^{t_{0}+\omega} b(s) e^{\int_{s}^{t_{0}+\omega} a(\tau) d \tau} d s}{1-e^{\int_{0}^{\omega} a(\tau) d \tau}}+\int_{t_{0}}^{t} b(s) e^{\int_{s}^{t} a(\tau) d \tau} d s \\
& =\frac{\int_{t_{0}}^{t_{0}+w} b(s) e^{\int_{s-\omega}^{t} a(\tau) d \tau} d s+\int_{t_{0}}^{t} b(s) e^{\int_{s}^{t} a(\tau) d \tau} d s-e^{\int_{0}^{\omega} a(\tau) d \tau} \int_{t_{0}}^{t} b(s) e^{\int_{s}^{t} a(\tau) d \tau} d s}{1-e^{\int_{0}^{\omega} a(\tau) d \tau}} \\
& =\frac{\int_{t_{0}}^{t_{0}+w} b(s) e^{\int_{s-\omega}^{t} a(\tau) d \tau} d s+\int_{t_{0}+\omega}^{t+\omega} b(s) e_{\int_{s-\omega}^{t} a(\tau) d \tau}^{t^{\omega}} d s-\int_{t_{0}}^{t} b(s) e^{\int_{s-\omega}^{t} a(\tau) d \tau} d s}{1-e_{0}^{\int_{0}^{\omega} a(\tau) d \tau}} \\
& =\frac{\int_{t}^{t+\omega} b(s) e_{\int_{s-\omega}^{t} a(\tau) d \tau}^{\int_{0}} d s}{1-e^{\int_{0} a(\tau) d \tau}} .
\end{aligned}
$$

When $m(a(t))<0$, it follows that

$$
\begin{aligned}
x(t) & =\frac{\int_{t}^{t+\omega} b(s) e^{\int_{s-\omega}^{t} a(\tau) d \tau} d s}{1-e^{\int_{0}^{\omega} a(\tau) d \tau}} \\
& =\frac{\int_{-\infty}^{t+\omega} b(s) e^{\int_{s-\omega}^{t} a(\tau) d \tau} d s-\int_{-\infty}^{t} b(s) e^{\int_{s-\omega}^{t} a(\tau) d \tau} d s}{1-e^{\int_{0}^{\omega} a(\tau) d \tau}} \\
& =\frac{\int_{-\infty}^{t} b(s) e^{\int_{s}^{t} a(\tau) d \tau} d s-\int_{-\infty}^{t} b(s) \int^{\int_{s-\omega}^{t} a(\tau) d \tau} d s}{1-e^{\int_{0}^{\omega} a(\tau) d \tau}} \\
& =\frac{\int_{-\infty}^{t} b(s) e^{\int_{s}^{t} a(\tau) d \tau} d s-e^{\int_{0}^{\omega} a(\tau) d \tau} \int_{-\infty}^{t} b(s) e^{\int_{s}^{t} a(\tau) d \tau} d s}{1-e^{\int_{0}^{\omega} a(\tau) d \tau}} \\
& =\int_{-\infty}^{t} b(s) e^{\int_{s}^{t} a(\tau) d \tau} d s,
\end{aligned}
$$

thus we have

$$
\begin{aligned}
x(t+\omega) & =\int_{-\infty}^{t+\omega} b(s) e^{\int_{s}^{t+\omega} a(\tau) d \tau} d s \\
& =\int_{-\infty}^{t} b(s) e^{\int_{s}^{t} a(\tau) d \tau} d s \\
& =x(t) .
\end{aligned}
$$

Hence, $x(t)$ is a unique $\omega$-periodic solution of (2.3); we rewrite it as follows:

$$
\eta(t)=\int_{-\infty}^{t} b(s) e^{t_{s}^{t} a(\tau) d \tau} d s .
$$



as follows:

Similarly, we can prove that when $m(a(t))>0,(2.3)$ has a unique $\omega$-periodic solution

$$
\eta(t)=-\int_{t}^{+\infty} b(s) e^{\int_{s}^{t} a(\tau) d \tau} d s
$$

Remark 2.4. Under the conditions of Lemma 2.3, all the infinite integrals above are convergent.

\section{A Sufficient Condition of Integrability of the First-Order Nonlinear Differential Equation}

In this section, we discuss the integrability of (1.1)

Theorem 3.1. Consider (1.1), where $a_{0}(t), a_{n}(t)$ are continuous functions defined on certain interval $I$, if there is a constant $\gamma$ such that the following conditions hold:

$$
\begin{aligned}
& \left(H_{1}\right) a_{n-1}(t)=(-1)^{n-1}(n-1) a_{0}(t)\left(\int_{\gamma}^{t} a_{n}(s) d s\right)^{n-1}, \\
& \left(H_{2}\right) a_{k}(t)=(-1)^{k} C_{n}^{k} a_{0}(t)\left(\int_{\gamma}^{t} a_{n}(s) d s\right)^{k}, k=1,2, \ldots, n-2,
\end{aligned}
$$

then

(i) the general solution of (1.1) can be written as follows:

$$
x(t)= \pm\left(e^{\int a_{n-1}(t) d t}\left(C-(n-1) \int a_{0}(t) e^{-\int a_{n-1}(t) d t} d t\right)\right)^{-1 /(n-1)}+\int_{\gamma}^{t} a_{n}(s) d s
$$

where $C$ is an integration constant which makes the number of root greater than zero if $n$ is an odd number.

(ii) Equation (1.1) has a particular solution

$$
\eta(t)=\int_{r}^{t} a_{n}(s) d s
$$

Proof. (i) Since the conditions $\left(H_{1}\right),\left(H_{2}\right)$ hold, (1.1) can be written as follows:

$$
\begin{aligned}
\frac{d\left(x-\int_{\gamma}^{t} a_{n}(s) d s\right)}{d t}= & \left(x-\int_{\gamma}^{t} a_{n}(s) d s\right) \\
& \times\left[(-1)^{n} a_{0}(t)\left(\int_{r}^{t} a_{n}(s) d s\right)^{n-1}+a_{0}(t)\left(x-\int_{\gamma}^{t} a_{n}(s) d s\right)^{n-1}\right] .
\end{aligned}
$$


Let $y(t)=x-\int_{\gamma}^{t} a_{n}(s) d s,(3.3)$ can be changed as follows:

$$
\frac{d y}{d t}=y\left[(-1)^{n} a_{0}(t)\left(\int_{r}^{t} a_{n}(s) d s\right)^{n-1}+a_{0}(t) y^{n-1}\right]
$$

This is the Bernoulli-type equation, set $u(t)=y^{1-n}(t)$, the Bernoulli-type equation (3.4) becomes

$$
\begin{aligned}
\frac{d u}{d t} & =(-1)^{n-1}(n-1) a_{0}(t)\left(\int_{r}^{t} a_{n}(s) d s\right)^{n-1} u-(n-1) a_{0}(t) \\
& =a_{n-1}(t) u-(n-1) a_{0}(t)
\end{aligned}
$$

according to the formula of general solution of linear differential equation, we can get that the general solution of (3.5) is given by

$$
u(t)=e^{\int a_{n-1}(t) d t}\left(C-(n-1) \int a_{0}(t) e^{-\int a_{n-1}(t) d t} d t\right) .
$$

By the variable transformation of $u(t)=y^{1-n}(t)$, it follows that

$$
y(t)= \pm\left(e^{\int a_{n-1}(t) d t}\left(C-(n-1) \int a_{0}(t) e^{-\int a_{n-1}(t) d t} d t\right)\right)^{-1 /(n-1)},
$$

since $y(t)=x-\int_{r}^{t} a_{n}(s) d s$, we can get

$$
x(t)= \pm\left(e^{\int a_{n-1}(t) d t}\left(C-(n-1) \int a_{0}(t) e^{-\int a_{n-1}(t) d t} d t\right)\right)^{-1 /(n-1)}+\int_{\gamma}^{t} a_{n}(s) d s
$$

Remark 3.2. If $n$ is an even number, take " + " in the front of above-corresponding formulas, if $n$ is an odd number, take " \pm ".

(ii) By (1.1), we set $f(t, x)=\sum_{k=0}^{n} a_{k}(t) x^{n-k}$, thus $f(t, x)$ is continuous on $I \times R, f_{x}(t, x)$ is also continuous on $I \times R$, hence (1.1) satisfies the principle of existence and uniqueness of differential equation, therefore, (1.1) has no singular solution.

From the variable transformation of $u(t)=y^{1-n}(t)$, it requires $y \neq 0$, but from (3.4), we know $y=0$ is indeed a solution of (3.4). Since $y=x-\int_{\gamma}^{t} a_{n}(s) d s$, substitute $\eta(t)=\int_{\gamma}^{t} a_{n}(s) d s$ into (1.1), it just satisfies (1.1), so it is a particular solution of (1.1).

This is the end of the proof of Theorem 3.1.

Corollary 3.3. Consider Abel type differential equation (1.2), $a(t), d(t)$ are continuous functions defined on certain interval $I$, if there is a constant $\gamma$ such that the following conditions hold:

$$
\begin{aligned}
& \left(H_{1}\right) b(t)=-3 a(t) \int_{\gamma}^{t} d(s) d s, \\
& \left(H_{2}\right) c(t)=2 a(t)\left(\int_{\gamma}^{t} d(s) d s\right)^{2},
\end{aligned}
$$


then the general solution of (1.2) can be written as follows:

$$
x(t)= \pm\left(e^{\int c(t) d t}\left(C-2 \int a(t) e^{-\int c(t) d t} d t\right)\right)^{-1 / 2}+\int_{\gamma}^{t} d(s) d s
$$

and $\int_{\gamma}^{t} d(s) d s$ is a particular solution of (1.2).

Corollary 3.4. Consider the following Ricatti's equation:

$$
\frac{d x}{d t}=a(t) x^{2}+b(t) x+c(t)
$$

$a(t), c(t)$ are continuous functions defined on certain interval $I$, if there is a constant $\gamma$ such that the following condition holds:

$$
\left(H_{1}\right) b(t)=-a(t) \int_{\gamma}^{t} c(s) d s,
$$

then the general solution of (3.10) can be written as follows:

$$
x(t)=\left(e^{\int b(t) d t}\left(C-\int a(t) e^{-\int b(t) d t} d t\right)\right)^{-1}+\int_{\gamma}^{t} c(s) d s,
$$

and $\eta(t)=\int_{\gamma}^{t} c(s) d s$ is a particular solution of (3.10).

\section{The Existence and Stability of Periodic Solutions of the First-Order Nonlinear Differential Equation}

Define

$$
\begin{array}{ll}
\Omega_{1}=\left\{x \mid x>\int_{\gamma}^{t_{0}} a_{n}(s) d s\right\}, & \Omega_{2}=\left\{x \mid x>\inf _{t \in[0, \omega]} \int_{\gamma}^{t} a_{n}(s) d s\right\}, \\
\Omega_{3}=\left\{x \mid x<\int_{\gamma}^{t_{0}} a_{n}(s) d s\right\}, & \Omega_{4}=\left\{x \mid x<\sup _{t \in[0, \omega]} \int_{\gamma}^{t} a_{n}(s) d s\right\},
\end{array}
$$

where $a_{n}(t), \gamma, \omega$ are the same function and numbers as the following Theorem 4.1 , and $t_{0}$ is any given initial time of (1.1).

Theorem 4.1. Consider (1.1), $n$ is an odd number, and $n>2, a_{0}(t), a_{n}(t)$ are continuous $\omega$-periodic functions defined on $R$, if there is a constant $\gamma$ such that the following conditions hold:

$$
\begin{aligned}
& \left(H_{1}\right) a_{n-1}(t)=(n-1) a_{0}(t)\left(\int_{\gamma}^{t} a_{n}(s) d s\right)^{n-1}, \\
& \left(H_{2}\right) a_{k}(t)=(-1)^{k} C_{n}^{k} a_{0}(t)\left(\int_{\gamma}^{t} a_{n}(s) d s\right)^{k}, k=1,2, \ldots, n-2, \\
& \left(H_{3}\right) m\left(a_{n}(t)\right)=0, a_{n}(t) \not \equiv 0, \\
& \left(H_{4}\right) a_{0}(t)<0,
\end{aligned}
$$


then

(i) equation (1.1) has three w-periodic solutions $\eta(t), \phi(t), \chi(t)$, and they can be written as follows:

$$
\begin{gathered}
\eta(t)=\int_{\gamma}^{t} a_{n}(s) d s \\
\phi(t)=\left(-(n-1) \int_{-\infty}^{t} a_{0}(s) e^{\int_{s}^{t} a_{n-1}(\tau) d \tau} d s\right)^{-1 /(n-1)}+\int_{\gamma}^{t} a_{n}(s) d s, \\
x(t)=-\left(-(n-1) \int_{-\infty}^{t} a_{0}(s) e^{\int_{s}^{t} a_{n-1}(\tau) d \tau} d s\right)^{-1 /(n-1)}+\int_{\gamma}^{t} a_{n}(s) d s
\end{gathered}
$$

(ii) if given any initial value $x\left(t_{0}\right) \in \Omega_{1}$, then the periodic solution $\phi(t)$ of (1.1) is globally attractive in $\Omega_{2}$;

(iii) if given any initial value $x\left(t_{0}\right) \in \Omega_{3}$, then the periodic solution $x(t)$ of (1.1) is globally attractive in $\Omega_{4}$.

Proof. (i) Since the conditions $\left(H_{1}\right),\left(H_{2}\right)$ hold, (1.1) can be written as follows:

$$
\begin{aligned}
\frac{d\left(x-\int_{\gamma}^{t} a_{n}(s) d s\right)}{d t}= & \left(x-\int_{\gamma}^{t} a_{n}(s) d s\right) \\
& \times\left[-a_{0}(t)\left(\int_{r}^{t} a_{n}(s) d s\right)^{n-1}+a_{0}(t)\left(x-\int_{r}^{t} a_{n}(s) d s\right)^{n-1}\right] .
\end{aligned}
$$

Let $y(t)=x-\int_{\gamma}^{t} a_{n}(s) d s,(4.3)$ can be changed as follows:

$$
\frac{d y}{d t}=y\left[-a_{0}(t)\left(\int_{r}^{t} a_{n}(s) d s\right)^{n-1}+a_{0}(t) y^{n-1}\right] .
$$

This is the Bernoulli-type equation, set $u(t)=y^{1-n}(t)$, the Bernoulli-type equation (4.4) becomes

$$
\begin{gathered}
\frac{d u}{d t}=(n-1) a_{0}(t)\left(\int_{r}^{t} a_{n}(s) d s\right)^{n-1} u-(n-1) a_{0}(t) \\
\frac{d u}{d t}=a_{n-1}(t) u-(n-1) a_{0}(t)
\end{gathered}
$$


By $\left(H_{3}\right),\left(H_{4}\right)$, it follows that

$$
\begin{aligned}
\int_{\gamma}^{t+\omega} a_{n}(s) d s & =\int_{\gamma}^{t} a_{n}(s) d s+\int_{t}^{t+\omega} a_{n}(s) d s \\
& =\int_{\gamma}^{t} a_{n}(s) d s+\int_{0}^{\omega} a_{n}(s) d s \\
& =\int_{\gamma}^{t} a_{n}(s) d s,
\end{aligned}
$$

thus $\int_{\gamma}^{t} a_{n}(s) d s$ is an $\omega$-periodic function, therefore, $(n-1) a_{0}(t)\left(\int_{\gamma}^{t} a_{n}(s) d s\right)^{n-1}$ is also an $\omega$ periodic function and

$$
a_{n-1}(t)=(n-1) a_{0}(t)\left(\int_{\gamma}^{t} a_{n}(s) d s\right)^{n-1} \leq 0,
$$

since $a_{0}(t)<0, a_{n}(t) \neq 0$ (not identically equal to zero), thus we have

$$
m\left(a_{n-1}(t)\right)<0,
$$

according to Lemma 2.3, it follows that (4.5) has a unique $\omega$-periodic solution as follows

$$
\varphi(t)=-(n-1) \int_{-\infty}^{t} a_{0}(s) e^{\int_{s}^{t} a_{n-1}(\tau) d \tau} d s,
$$

from (4.9), it is easy to know that $\varphi(t)>0$, since $\varphi(t)$ is periodic on $R$, it is bounded on $R$, so it is positive and bounded, since $u(t)=y^{1-n}(t)$, and by Lemmas 2.1 and 2.2, it follows that (4.4) has two $\omega$-periodic solutions as follows:

$$
\begin{gathered}
\psi(t)=\left(-(n-1) \int_{-\infty}^{t} a_{0}(s) e^{\int_{s}^{t} a_{n-1}(\tau) d \tau} d s\right)^{-1 /(n-1)} \quad\left(\psi\left(t_{0}\right)>0\right), \\
\psi^{*}(t)=-\left(-(n-1) \int_{-\infty}^{t} a_{0}(s) e^{\int_{s}^{t} a_{n-1}(\tau) d \tau} d s\right)^{-1 /(n-1)} \quad\left(\psi^{*}\left(t_{0}\right)<0\right),
\end{gathered}
$$


thus $\psi(t)$ is also positive and bounded, and $\psi^{*}(t)$ is negative and bounded, by virtue of $y(t)=$ $x-\int_{\gamma}^{t} a_{n}(s) d s,(1.1)$ has two $\omega$-periodic solutions as follows:

$$
\begin{gathered}
\phi(t)=\left(-(n-1) \int_{-\infty}^{t} a_{0}(s) e^{\int_{s}^{t} a_{n-1}(\tau) d \tau} d s\right)^{-1 /(n-1)}+\int_{\gamma}^{t} a_{n}(s) d s, \\
X(t)=-\left(-(n-1) \int_{-\infty}^{t} a_{0}(s) e^{\int_{s}^{t} a_{n-1}(\tau) d \tau} d s\right)^{-1 /(n-1)}+\int_{\gamma}^{t} a_{n}(s) d s,
\end{gathered}
$$

and $\phi\left(t_{0}\right) \in \Omega_{1}, \phi(t) \in \Omega_{2}, \chi\left(t_{0}\right) \in \Omega_{3}, \chi(t) \in \Omega_{4}$.

By (1.1), we set $f(t, x)=\sum_{k=0}^{n} a_{k}(t) x^{n-k}$, thus $f(t, x)$ is continuous on $R \times R$, and $f_{x}(t, x)$ is also continuous on $R \times R$, hence (1.1) satisfies the principle of existence and uniqueness of differential equation, therefore, (1.1) has no singular solution.

From the variable transformation of $u(t)=y^{1-n}(t)$, it requires $y \neq 0$, but from (4.4), we know $y=0$ is indeed a solution of (4.4), since $y(t)=x-\int_{\gamma}^{t} a_{n}(s) d s$, hence $\eta(t)=\int_{\gamma}^{t} a_{n}(s) d s$ is a periodic solution of (1.1). Substitute $\eta(t)=\int_{\gamma}^{t} a_{n}(s) d s$ into (1.1), it just satisfies (1.1), so $\eta(t)=\int_{\gamma}^{t} a_{n}(s) d s$ is indeed a periodic particular solution of (1.1), therefore, we have proved that (1.1) has three $\omega$-periodic solutions $\eta(t), \phi(t)$, and $\chi(t)$.

(ii) According to the theories of linear differential equation, we know that the unique solution $u(t)$ of (4.5) with positive initial value $u\left(t_{0}\right)=u_{0}$ is given by

$$
u(t)=u_{0} e^{\int_{t_{0}}^{t} a_{n-1}(\tau) d \tau}-(n-1) \int_{t_{0}}^{t} a_{0}(s) e^{\int_{s}^{t} a_{n-1}(\tau) d \tau} d s
$$

Since

$$
a_{n-1}(t)=(n-1) a_{0}(t)\left(\int_{\gamma}^{t} a_{n}(\tau) d \tau\right)^{n-1}
$$

thus $a_{n-1}(t)$ is an $\omega$-periodic function on $R$, and by (4.7), we know $a_{n-1}(t) \leq 0$, thus $\left(a_{n-1}\right)_{L}<$ 0 .

Following we prove that there is a positive number $T>t_{0}$, such that

$$
(n-1) \frac{a_{0_{M}}}{\left(a_{n-1}\right)_{L}}(1-\varepsilon) \leq u(t) \leq u_{0}-\frac{2 \omega a_{0_{L}}}{1-\int_{0}^{\omega} a_{n-1}(\tau) d \tau}
$$

as $t>T$, here $\varepsilon$ is any small positive number and $\varepsilon<1$. 
From (4.12), given any positive number $u_{0}>0$, by the condition $a_{0}(t)<0$ of Theorem 4.1, it follows that $u(t)>0$; following we first prove that $u(t)$ has upper bound on $\left[t_{0},+\infty\right)$

$$
\begin{aligned}
u(t) & =u_{0} e^{\int_{t_{0}}^{t} a_{n-1}(\tau) d \tau}-(n-1) \int_{t_{0}}^{t} a_{0}(s) e^{\int_{s}^{t} a_{n-1}(\tau) d \tau} d s \\
& \leq u_{0}-(n-1) \int_{-\infty}^{t} a_{0}(s) e^{\int_{s}^{t} a_{n-1}(\tau) d \tau} d s .
\end{aligned}
$$

According to Lemma 2.3, it follows that

$$
\begin{aligned}
u(t) & \leq u_{0}-(n-1) \int_{-\infty}^{t} a_{0}(s) e^{\int_{s}^{t} a_{n-1}(\tau) d \tau} d s \\
& =u_{0}-\frac{(n-1) \int_{t}^{t+\omega} a_{0}(s) e^{\int_{s-\omega}^{t} a_{n-1}(\tau) d \tau} d s}{1-e^{\int_{0}^{\omega} a_{n-1}(\tau) d \tau}} \\
& =u_{0}-\frac{\omega(n-1) a_{0}(\xi) e^{\int_{\xi-\omega}^{t} a_{n-1}(\tau) d \tau}}{1-e^{\int_{0}^{\omega} a_{n-1}(\tau) d \tau}, \quad(t<\xi<t+\omega)} \\
& =u_{0}-\frac{\omega(n-1) a_{0}(\xi) e^{\int_{\xi}^{t} a_{n-1}(\tau) d \tau} e^{\int_{0}^{\omega} a_{n-1}(\tau) d \tau}}{1-e^{\int_{0}^{\omega} a_{n-1}(\tau) d \tau}} \\
& \leq u_{0}-\frac{\omega(n-1) a_{0}(\xi) e^{\int_{0}^{\omega}\left|a_{n-1}(\tau)\right| d \tau} e^{\int_{0}^{\omega} a_{n-1}(\tau) d \tau}}{1-e^{\int_{0}^{\omega} a_{n-1}(\tau) d \tau}} \\
& =u_{0}-\frac{\omega(n-1) a_{0}(\xi) e^{\int_{0}^{\omega}\left(\left|a_{n-1}(\tau)\right|+a_{n-1}(\tau)\right) d \tau}}{1-e^{\int_{0}^{\omega} a_{n-1}(\tau) d \tau}} \\
& =u_{0}-\frac{\omega(n-1) a_{0}(\xi)}{1-e_{0}^{\int_{0}^{\omega} a_{n-1}(\tau) d \tau}} \\
& \leq u_{0}-\frac{\omega(n-1) a_{0_{L}}}{1-e^{\int_{0}^{\omega} a_{n-1}(\tau) d \tau}} .
\end{aligned}
$$

So $u(t)$ has upper bound on $\left[t_{0},+\infty\right)$.

Secondly, we prove that $u(t) \geq(n-1)\left(a_{0_{M}} /\left(a_{n-1}\right)_{L}\right)(1-\varepsilon)$ as $t>T$

$$
\begin{aligned}
u(t) & =u_{0} e^{\int_{t_{0}}^{t} a_{n-1}(\tau) d \tau}-(n-1) \int_{t_{0}}^{t} a_{0}(s) e^{\int_{s}^{t} a_{n-1}(\tau) d \tau} d s \\
& \geq-(n-1) \int_{t_{0}}^{t} a_{0}(s) e^{\int_{s}^{t} a_{n-1}(\tau) d \tau} d s \\
& \geq-(n-1) \int_{t_{0}}^{t} a_{0_{M}} e^{\left(a_{n-1}\right)_{L}(t-s)} d s
\end{aligned}
$$




$$
\begin{aligned}
& =-(n-1)\left(-\frac{a_{0_{M}}}{\left(a_{n-1}\right)_{L}}\right)\left(1-e^{\left(a_{n-1}\right)_{L}\left(t-t_{0}\right)}\right) \\
& =(n-1) \frac{a_{0_{M}}}{\left(a_{n-1}\right)_{L}}\left(1-e^{\left(a_{n-1}\right)_{L}\left(t-t_{0}\right)}\right) .
\end{aligned}
$$

Since $\lim _{t \rightarrow+\infty} e^{\left(a_{n-1}\right)_{L}\left(t-t_{0}\right)}=0$, forall $\varepsilon>0(\varepsilon<1)$, there must be $T>t_{0}$, when $t>T$, we have $e^{\left(a_{n-1}\right)_{L}\left(t-t_{0}\right)}<\varepsilon$, hence when $t>T$, we can get

$$
u(t) \geq(n-1) \frac{a_{0_{M}}}{\left(a_{n-1}\right)_{L}}(1-\varepsilon),
$$

therefore, we have proved that there is a positive number $T>t_{0}$, as $t>T$, it follows that

$$
(n-1) \frac{a_{0_{M}}}{\left(a_{n-1}\right)_{L}}(1-\varepsilon) \leq u(t) \leq u_{0}-\frac{\omega(n-1) a_{0_{L}}}{1-e^{\int_{0}^{\omega} a_{n-1}(\tau) d \tau}}, \quad(t>T),
$$

under the transformation of $u(t)=y^{1-n}(t), y(t)=x(t)-\int_{\gamma}^{t} a_{n}(s) d s$, we can get the following. If given the initial value

$$
x\left(t_{0}\right)=\left(u_{0}\right)^{-1 /(n-1)}+\int_{\gamma}^{t_{0}} a_{n}(s) d s \in \Omega_{1},
$$

then the unique solution $x(t)$ of (1.1) is given by

$$
x(t)=\left(u_{0} e^{\int_{t_{0}}^{t} a_{n-1}(\tau) d \tau}-(n-1) \int_{t_{0}}^{t} a_{0}(s) e^{t_{s}^{t} a_{n-1}(\tau) d \tau} d s\right)^{-1 /(n-1)}+\int_{\gamma}^{t} a_{n}(s) d s,
$$

if given the initial value

$$
x\left(t_{0}\right)=-\left(u_{0}\right)^{-1 /(n-1)}+\int_{\gamma}^{t_{0}} a_{n}(s) d s \in \Omega_{3}
$$

then the unique solution $x^{*}(t)$ of (1.1) is given by

$$
x^{*}(t)=-\left(u_{0} e^{\int_{t_{0}}^{t} a_{n-1}(\tau) d \tau}-(n-1) \int_{t_{0}}^{t} a_{0}(s) e^{\int_{s}^{t} a_{n-1}(\tau) d \tau} d s\right)^{-1 /(n-1)}+\int_{\gamma}^{t} a_{n}(s) d s,
$$

and it is easy to know $x(t) \in \Omega_{2}$ and $x^{*}(t) \in \Omega_{4}$, respectively.

Define a Lyapunov function as follows:

$$
V(t, u, \varphi)=(u-\varphi)^{2}
$$


where $u(t)$ is the unique solution with the positive initial value $u\left(t_{0}\right)=u_{0}$ of (4.5), and $\varphi$ is the unique positive $\omega$-periodic solution of (4.5), differentiating both sides of (4.24) along the solution of (4.5), we get

$$
\begin{aligned}
\frac{d V(t, u, \varphi)}{d t} & =2(u-\varphi)\left(\frac{d u}{d t}-\frac{d \varphi}{d t}\right) \\
& =2 a_{n-1}(t)(u-\varphi)^{2},
\end{aligned}
$$

by (4.24), we have

$$
\frac{d V(t, u, \varphi)}{V(t, u, \varphi)}=2 a_{n-1}(t) d t
$$

thus

$$
V(t, u, \varphi)=V\left(t_{0}, u\left(t_{0}\right), \varphi\left(t_{0}\right)\right) e^{\int_{t_{0}}^{t} 2 a_{n-1}(s) d s},
$$

by (4.24), we can get

$$
(u-\varphi)^{2}=\left(u\left(t_{0}\right)-\varphi\left(t_{0}\right)\right)^{2} e^{\int_{t_{0}}^{t} 2 a_{n-1}(s) d s},
$$

by (4.28), that it follows

$$
|u-\varphi|=\left|u\left(t_{0}\right)-\varphi\left(t_{0}\right)\right| e^{\int_{t_{0}}^{t} a_{n-1}(s) d s}
$$

since

$$
x(t)=u^{-1 /(n-1)}(t)+\int_{\gamma}^{t} a_{n}(s) d s, \quad \phi(t)=\varphi^{-1 /(n-1)}(t)+\int_{\gamma}^{t} a_{n}(s) d s,
$$

it follows that

$$
u(t)=\frac{1}{\left(x(t)-\int_{\gamma}^{t} a_{n}(s) d s\right)^{n-1}}, \quad \varphi(t)=\frac{1}{\left(\phi(t)-\int_{\gamma}^{t} a_{n}(s) d s\right)^{n-1}},
$$


substitute (4.31) into (4.29), it follows that

$$
\begin{aligned}
& \left|\frac{1}{\left(x(t)-\int_{\gamma}^{t} a_{n}(s) d s\right)^{n-1}}-\frac{1}{\left(\phi(t)-\int_{\gamma}^{t} a_{n}(s) d s\right)^{n-1}}\right| \\
& \quad=\left|\frac{1}{\left(x\left(t_{0}\right)-\int_{\gamma}^{t_{0}} a_{n}(s) d s\right)^{n-1}}-\frac{1}{\left(\phi\left(t_{0}\right)-\int_{\gamma}^{t_{0}} a_{n}(s) d s\right)^{n-1}}\right| e^{\int_{t_{0}}^{t} a_{n-1}(s) d s} .
\end{aligned}
$$

According to mean value theorem, we can get

$$
\left|-(n-1) \xi^{-n}(x(t)-\phi(t))\right|=\left|-(n-1) \zeta^{-n}\left(x\left(t_{0}\right)-\phi\left(t_{0}\right)\right)\right| e^{\int_{t_{0}}^{t} a_{n-1}(s) d s},
$$

where

$$
x(t)-\int_{\gamma}^{t} a_{n}(s) d s<\xi<\phi(t)-\int_{\gamma}^{t} a_{n}(s) d s
$$

or

$$
\begin{gathered}
\phi(t)-\int_{\gamma}^{t} a_{n}(s) d s<\xi<x(t)-\int_{\gamma}^{t} a_{n}(s) d s, \\
x\left(t_{0}\right)-\int_{\gamma}^{t_{0}} a_{n}(s) d s<\zeta<\phi\left(t_{0}\right)-\int_{\gamma}^{t_{0}} a_{n}(s) d s
\end{gathered}
$$

or

$$
\phi\left(t_{0}\right)-\int_{\gamma}^{t_{0}} a_{n}(s) d s<\zeta<x\left(t_{0}\right)-\int_{\gamma}^{t_{0}} a_{n}(s) d s .
$$

Since $\varphi(t)$ is positive and bounded, and from (4.19), (4.31), we know that

$$
x(t)-\int_{\gamma}^{t} a_{n}(s) d s, \phi(t)-\int_{\gamma}^{t} a_{n}(s) d s, x\left(t_{0}\right)-\int_{\gamma}^{t_{0}} a_{n}(s) d s, \phi\left(t_{0}\right)-\int_{\gamma}^{t_{0}} a_{n}(s) d s
$$

are positive and bounded as $t>T$, hence $\xi$, $\zeta$ are positive and bounded as $t>T$, so we have

$$
|x(t)-\phi(t)|=\left(\frac{\xi}{\zeta}\right)^{n}\left|x\left(t_{0}\right)-\phi\left(t_{0}\right)\right| e^{\int_{t_{0}}^{t} a_{n-1}(s) d s}
$$


by (4.8), it follows that

$$
\int_{t_{0}}^{t} a_{n-1}(s) d s \longrightarrow-\infty
$$

as $t \rightarrow+\infty$, and by (4.38), we can get that the $\omega$-periodic solution $\phi(t)$ of $(1.1)$ is globally attractive in $\Omega_{2}$.

(iii) The proof of the periodic solution $\chi(t)$ of (1.1) being globally attractive in $\Omega_{4}$ is similar to that of the periodic solution $\phi(t)$ of (1.1) being globally attractive in $\Omega_{2}$, so we omit it here.

This is the end of the proof of Theorem 4.1.

Remark 4.2. In Theorem 4.1, since the transformation of $u=y^{1-n}$, it follows $u>0$, thus we suppose the initial value $u\left(t_{0}\right)>0$.

Theorem 4.3. Consider (1.1), $n$ is an even number, and $n \geq 2, a_{0}(t), a_{n}(t)$ are continuous $\omega$-periodic functions defined on $R$, if there is a constant $\gamma$ such that the following conditions hold:

$$
\begin{aligned}
& \left(H_{1}\right) a_{n-1}(t)=(1-n) a_{0}(t)\left(\int_{\gamma}^{t} a_{n}(s) d s\right)^{n-1}, \\
& \left(H_{2}\right) a_{k}(t)=(-1)^{k} C_{n}^{k} a_{0}(t)\left(\int_{\gamma}^{t} a_{n}(s) d s\right)^{k}, k=1,2, \ldots, n-2, \\
& \left(H_{3}\right) m\left(a_{n}(t)\right)=0, a_{n}(t) \not \equiv 0, \\
& \left(H_{4}\right) a_{0}(t)<0, \int_{\gamma}^{t} a_{n}(s) d s \leq 0,
\end{aligned}
$$

then

(i) equation (1.1) has two w-periodic solutions $\eta(t), \phi(t)$, and they can be written as follows:

$$
\begin{gathered}
\eta(t)=\int_{\gamma}^{t} a_{n}(s) d s \\
\phi(t)=\left(-(n-1) \int_{-\infty}^{t} a_{0}(s) e^{\int_{s}^{t} a_{n-1}(\tau) d \tau} d s\right)^{-1 /(n-1)}+\int_{\gamma}^{t} a_{n}(s) d s .
\end{gathered}
$$

(ii) If given any initial value $x\left(t_{0}\right) \in \Omega_{1}=\left\{x \mid x>\int_{\gamma}^{t_{0}} a_{n}(s) d s\right\}$, then the periodic solution $\phi(t)$ of (1.1) is globally attractive in $\Omega_{2}=\left\{x \mid x>\inf _{t \in[0, \omega]} \int_{\gamma}^{t} a_{n}(s) d s\right\}$.

Proof. The proof of Theorem 4.3 is similar to that of Theorem 4.1, so we omit it here.

Theorem 4.4. Consider (1.1), $n$ is an even number, and $n \geq 2, a_{0}(t), a_{n}(t)$ are continuous $\omega$-periodic functions defined on $R$, if there is a constant $\gamma$ such that the following conditions hold:

$$
\begin{aligned}
& \left(H_{1}\right) a_{n-1}(t)=(1-n) a_{0}(t)\left(\int_{\gamma}^{t} a_{n}(s) d s\right)^{n-1}, \\
& \left(H_{2}\right) a_{k}(t)=(-1)^{k} C_{n}^{k} a_{0}(t)\left(\int_{\gamma}^{t} a_{n}(s) d s\right)^{k}, k=1,2, \ldots, n-2, \\
& \left(H_{3}\right) m\left(a_{n}(t)\right)=0, a_{n}(t) \not \equiv 0, \\
& \left(H_{4}\right) a_{0}(t)>0, \int_{\gamma}^{t} a_{n}(s) d s \geq 0,
\end{aligned}
$$


then

(i) equation (1.1) has two w-periodic solutions $\eta(t), \phi(t)$, and they can be written as follows:

$$
\begin{gathered}
\eta(t)=\int_{\gamma}^{t} a_{n}(s) d s \\
\phi(t)=\left(-(n-1) \int_{-\infty}^{t} a_{0}(s) e^{\int_{s}^{t} a_{n-1}(\tau) d \tau} d s\right)^{-1 /(n-1)}+\int_{\gamma}^{t} a_{n}(s) d s .
\end{gathered}
$$

(ii) If given any initial value $x\left(t_{0}\right) \in \Omega_{1}=\left\{x \mid x<\int_{\gamma}^{t_{0}} a_{n}(s) d s\right\}$, then the periodic solution $\phi(t)$ of (1.1) is globally attractive in $\Omega_{2}=\left\{x \mid x<\sup _{t \in[0, \omega]} \int_{\gamma}^{t} a_{n}(s) d s\right\}$.

Proof. The proof of Theorem 4.4 is similar to that of Theorem 4.1, so we omit it here.

From Theorem 4.1 , if $n=3$, it is easy for us to draw the following corollary.

Define

$$
\begin{array}{ll}
\Omega_{1}=\left\{x \mid x>\int_{\gamma}^{t_{0}} d(s) d s\right\}, & \Omega_{2}=\left\{x \mid x>\inf _{t \in[0, \omega]} \int_{\gamma}^{t} d(s) d s\right\}, \\
\Omega_{3}=\left\{x \mid x<\int_{\gamma}^{t_{0}} d(s) d s\right\}, & \Omega_{4}=\left\{x \mid x<\sup _{t \in[0, \omega]} \int_{\gamma}^{t} d(s) d s\right\},
\end{array}
$$

where $d(t), \gamma, \omega$ are the same function and numbers as the following Corollary 4.5 , and $t_{0}$ is any given initial time of (1.2).

Corollary 4.5. Consider Abel type differential equation (1.2), $a(t), d(t)$ are $\omega$-periodic continuous functions defined on $R$, if there is a constant $\gamma$ such that the following conditions hold:

$$
\begin{aligned}
& \left(H_{1}\right) b(t)=-3 a(t) \int_{\gamma}^{t} d(s) d s ; \\
& \left(H_{2}\right) c(t)=2 a(t)\left(\int_{\gamma}^{t} d(s) d s\right)^{2} ; \\
& \left(H_{3}\right) m(d(t))=0, d(t) \not \equiv 0 ; \\
& \left(H_{4}\right) a(t)<0 ;
\end{aligned}
$$

then

(i) equation (1.2) has three $\omega$-periodic solutions $\eta(t), \phi(t), \chi(t)$, and they can be written as follows:

$$
\begin{gathered}
\eta(t)=\int_{\gamma}^{t} d(s) d s, \\
\phi(t)=\left(-2 \int_{-\infty}^{t} a(s) e^{\int_{s}^{t} c(\tau) d \tau} d s\right)^{-1 / 2}+\int_{\gamma}^{t} d(s) d s, \\
x(t)=-\left(-2 \int_{-\infty}^{t} a(s) e^{\int_{s}^{t} c(\tau) d \tau} d s\right)^{-1 / 2}+\int_{\gamma}^{t} d(s) d s .
\end{gathered}
$$


(ii) If given any initial value $x\left(t_{0}\right) \in \Omega_{1}$, then the periodic solution $\phi(t)$ of (1.2) is globally attractive in $\Omega_{2}$.

(iii) If given any initial value $x\left(t_{0}\right) \in \Omega_{3}$, then the periodic solution $x(t)$ of (1.2) is globally attractive in $\Omega_{4}$.

From Theorems 4.3 and 4.4 , if $n=2$, it is easy for us to draw the following corollaries.

Corollary 4.6. Consider Ricatti's equation (3.10), $a(t), c(t)$ are w-periodic continuous functions defined on $R$, if there is a constant $\gamma$ such that the following conditions hold:

$$
\begin{aligned}
& \left(H_{1}\right) b(t)=-a(t) \int_{r}^{t} c(s) d s ; \\
& \left(H_{2}\right) m(c(t))=0, c(t) \not \equiv 0 ; \\
& \left(H_{3}\right) a(t)<0, \int_{r}^{t} c(s) d s \leq 0 ;
\end{aligned}
$$

then

(i) equation (3.10) has two w-periodic solutions $\eta(t), \phi(t)$, and they can be written as follows:

$$
\begin{gathered}
\eta(t)=\int_{\gamma}^{t} c(s) d s \\
\phi(t)=\left(-\int_{-\infty}^{t} a(s) e^{\int_{s}^{t} b(\tau) d \tau} d s\right)^{-1}+\int_{\gamma}^{t} c(s) d s .
\end{gathered}
$$

(ii) If given any initial value $x\left(t_{0}\right) \in \Omega_{1}=\left\{x \mid x>\int_{\gamma}^{t_{0}} c(s) d s\right\}$, then the periodic solution $\phi(t)$ of (3.10) is globally attractive in $\Omega_{2}=\left\{x \mid x>\inf _{t \in[0, \omega]} \int_{\gamma}^{t} c(s) d s\right\}$.

Corollary 4.7. Consider Ricatti's equation (3.10) $a(t), c(t)$ are w-periodic continuous functions defined on $R$, if there is a constant $\gamma$ such that the following conditions hold:

$\left(H_{1}\right) b(t)=-a(t) \int_{\gamma}^{t} c(s) d s ;$

$\left(H_{2}\right) m(c(t))=0, c(t) \not \equiv 0$;

$\left(H_{3}\right) a(t)>0, \int_{\gamma}^{t} c(s) d s \geq 0 ;$

then,

(i) equation (3.10) has two w-periodic solutions $\eta(t), \phi(t)$, and they can be written as follows:

$$
\begin{gathered}
\eta(t)=\int_{\gamma}^{t} c(s) d s \\
\phi(t)=\left(-\int_{-\infty}^{t} a(s) e^{\int_{s}^{t} b(\tau) d \tau} d s\right)^{-1}+\int_{\gamma}^{t} c(s) d s .
\end{gathered}
$$

(ii) If given any initial value $x\left(t_{0}\right) \in \Omega_{1}=\left\{x \mid x<\int_{\gamma}^{t_{0}} c(s) d s\right\}$, then the periodic solution $\phi(t)$ of (3.10) is globally attractive in $\Omega_{2}=\left\{x \mid x<\sup _{t \in[0, \omega]} \int_{\gamma}^{t} c(s) d s\right\}$. 
Theorem 4.8. Consider (1.1), $n$ is an odd number, and $n>2, a_{0}(t), a_{n}(t)$ are continuous $\omega$-periodic functions defined on $R$, if there is a constant $\gamma$ such that the following conditions hold:

$$
\begin{aligned}
& \left(H_{1}\right) a_{n-1}(t)=(n-1) a_{0}(t)\left(\int_{\gamma}^{t} a_{n}(s) d s\right)^{n-1} ; \\
& \left(H_{2}\right) a_{k}(t)=(-1)^{k} C_{n}^{k} a_{0}(t)\left(\int_{\gamma}^{t} a_{n}(s) d s\right)^{k}, k=1,2, \ldots, n-2 ; \\
& \left(H_{3}\right) m\left(a_{n}(t)\right)=0, a_{n}(t) \not \equiv 0 ; \\
& \left(H_{4}\right) a_{0}(t)>0 ;
\end{aligned}
$$

then,

(i) equation (1.1) has three w-periodic solutions $\eta(t), \phi(t), \chi(t)$, and they can be written as follows:

$$
\begin{gathered}
\eta(t)=\int_{\gamma}^{t} a_{n}(s) d s \\
\phi(t)=\left((n-1) \int_{t}^{+\infty} a_{0}(s) e^{\int_{s}^{t} a_{n-1}(\tau) d \tau} d s\right)^{-1 /(n-1)}+\int_{\gamma}^{t} a_{n}(s) d s \\
x(t)=-\left((n-1) \int_{t}^{+\infty} a_{0}(s) e^{\int_{s}^{t} a_{n-1}(\tau) d \tau} d s\right)^{-1 /(n-1)}+\int_{\gamma}^{t} a_{n}(s) d s .
\end{gathered}
$$

(ii) if given any initial value $x\left(t_{0}\right) \in \Omega_{1} \cup \Omega_{2}$, then the periodic solution $\eta(t)$ of (1.1) is globally attractive in $\Omega_{3} \cup \Omega_{4}$, where

$$
\begin{aligned}
\Omega_{1} & =\left\{x \mid \int_{\gamma}^{t_{0}} a_{n}(s) d s \leq x<\left(\varphi\left(t_{0}\right)\right)^{-1 / n-1}+\int_{\gamma}^{t_{0}} a_{n}(s) d s\right\}, \\
\Omega_{2} & =\left\{x \mid \int_{\gamma}^{t_{0}} a_{n}(s) d s \geq x>-\left(\varphi\left(t_{0}\right)\right)^{-1 / n-1}+\int_{\gamma}^{t_{0}} a_{n}(s) d s\right\}, \\
\Omega_{3} & =\left\{x \mid \inf _{t \in[0, \omega]} \int_{\gamma}^{t} a_{n}(s) d s \leq x<\sup _{t \in[0, \omega]}\left((\varphi(t))^{-1 /(n-1)}+\int_{\gamma}^{t} a_{n}(s) d s\right)\right\}, \\
\Omega_{4} & =\left\{x \mid \sup _{t \in[0, \omega]} \int_{\gamma}^{t} a_{n}(s) d s \geq x>\inf _{t \in[0, \omega]}\left(-(\varphi(t))^{-1 /(n-1)}+\int_{\gamma}^{t} a_{n}(s) d s\right)\right\}, \\
\varphi(t) & =(n-1) \int_{t}^{+\infty} a_{0}(s) e^{\int_{s}^{t} a_{n-1}(\tau) d \tau} d s .
\end{aligned}
$$

Remark 4.9. Where $\varphi(t)$ above is the same function as that of the following formula (4.54), and $t_{0}$ is any given initial time of (1.1). 
Proof. (i) Since the conditions $\left(H_{1}\right),\left(H_{2}\right)$ hold, (1.1) can be written as follows:

$$
\begin{aligned}
\frac{d\left(x-\int_{\gamma}^{t} a_{n}(s) d s\right)}{d t}= & \left(x-\int_{\gamma}^{t} a_{n}(s) d s\right) \\
& \times\left[-a_{0}(t)\left(\int_{r}^{t} a_{n}(s) d s\right)^{n-1}+a_{0}(t)\left(x-\int_{\gamma}^{t} a_{n}(s) d s\right)^{n-1}\right] .
\end{aligned}
$$

Let $y(t)=x-\int_{\gamma}^{t} a_{n}(s) d s,(4.48)$ can be changed as follows:

$$
\frac{d y}{d t}=y\left[-a_{0}(t)\left(\int_{r}^{t} a_{n}(s) d s\right)^{n-1}+a_{0}(t) y^{n-1}\right] .
$$

This is the Bernoulli-type equation, set $u(t)=y^{1-n}(t)$, the Bernoulli-type equation (4.49) becomes

$$
\begin{gathered}
\frac{d u}{d t}=(n-1) a_{0}(t)\left(\int_{r}^{t} a_{n}(s) d s\right)^{n-1} u-(n-1) a_{0}(t), \\
\frac{d u}{d t}=a_{n-1}(t) u-(n-1) a_{0}(t) .
\end{gathered}
$$

By $\left(H_{3}\right),\left(H_{4}\right)$, it follows that

$$
\begin{aligned}
\int_{\gamma}^{t+\omega} a_{n}(s) d s & =\int_{\gamma}^{t} a_{n}(s) d s+\int_{t}^{t+\omega} a_{n}(s) d s \\
& =\int_{\gamma}^{t} a_{n}(s) d s+\int_{0}^{\omega} a_{n}(s) d s \\
& =\int_{\gamma}^{t} a_{n}(s) d s,
\end{aligned}
$$

thus $\int_{\gamma}^{t} a_{n}(s) d s$ is an $\omega$-periodic function, therefore, $(n-1) a_{0}(t)\left(\int_{\gamma}^{t} a_{n}(s) d s\right)^{n-1}$ is also an $\omega$ periodic function and

$$
a_{n-1}(t)=(n-1) a_{0}(t)\left(\int_{\gamma}^{t} a_{n}(s) d s\right)^{n-1} \geq 0,
$$

since $a_{0}(t)>0, a_{n}(t) \neq 0$ (not identically equal to zero), thus we have

$$
m\left(a_{n-1}(t)\right)>0,
$$


according to Lemma 2.3, it follows that (4.50) has a unique $\omega$-periodic solution as follows:

$$
\varphi(t)=(n-1) \int_{t}^{+\infty} a_{0}(s) e^{\int_{s}^{t} a_{n-1}(\tau) d \tau} d s
$$

from (4.54), it is easy to know that $\varphi(t)>0$, since $\varphi(t)$ is periodic on $R$, it is bounded on $R$, so it is positive and bounded, since $u(t)=y^{1-n}(t)$, and by Lemmas 2.1 and 2.2, it follows that (4.49) has two $\omega$-periodic solutions as follows:

$$
\begin{gathered}
\psi(t)=\left((n-1) \int_{t}^{+\infty} a_{0}(s) e^{\int_{s}^{t} a_{n-1}(\tau) d \tau} d s\right)^{-1 /(n-1)}, \quad\left(\psi\left(t_{0}\right)>0\right), \\
\psi^{*}(t)=-\left((n-1) \int_{t}^{+\infty} a_{0}(s) e^{\int_{s}^{t} a_{n-1}(\tau) d \tau} d s\right)^{-1 /(n-1)}, \quad\left(\psi^{*}\left(t_{0}\right)<0\right),
\end{gathered}
$$

thus $\psi(t)$ is also positive and bounded, and $\psi^{*}(t)$ is negative and bounded, by virtue of $y(t)=$ $x-\int_{\gamma}^{t} a_{n}(s) d s,(1.1)$ has two $\omega$-periodic solutions as follows:

$$
\begin{gathered}
\phi(t)=\left((n-1) \int_{t}^{+\infty} a_{0}(s) e^{\int_{s}^{t} a_{n-1}(\tau) d \tau} d s\right)^{-1 /(n-1)}+\int_{\gamma}^{t} a_{n}(s) d s, \\
x(t)=-\left((n-1) \int_{t}^{+\infty} a_{0}(s) e^{\int_{s}^{t} a_{n-1}(\tau) d \tau} d s\right)^{-1 /(n-1)}+\int_{\gamma}^{t} a_{n}(s) d s .
\end{gathered}
$$

By (1.1), we set $f(t, x)=\sum_{k=0}^{n} a_{k}(t) x^{n-k}$, thus $f(t, x)$ is continuous on $R \times R$, and $f_{x}(t, x)$ is also continuous on $R \times R$, hence (1.1) satisfies the principle of existence and uniqueness of differential equation, therefore, (1.1) has no singular solution.

From the variable transformation of $u(t)=y^{1-n}(t)$, it requires $y \neq 0$, but from (4.49), we know $y=0$ is indeed a solution of (4.49), since $y(t)=x-\int_{\gamma}^{t} a_{n}(s) d s$, hence $\eta(t)=\int_{\gamma}^{t} a_{n}(s) d s$ is a periodic solution of (1.1). Substitute $\eta(t)=\int_{\gamma}^{t} a_{n}(s) d s$ into (1.1), it just satisfies (1.1), so $\eta(t)=\int_{\gamma}^{t} a_{n}(s) d s$ is indeed a periodic particular solution of (1.1), therefore, we have proved that (1.1) has three $\omega$-periodic solutions $\eta(t), \phi(t)$ and $\chi(t)$.

(ii) According to the theories of linear differential equation, we know that the unique solution $u(t)$ of (4.50) with the positive initial value $u\left(t_{0}\right)=u_{0}$ is given by

$$
\begin{aligned}
u(t) & =u_{0} e^{\int_{t_{0}}^{t} a_{n-1}(\tau) d \tau}-(n-1) \int_{t_{0}}^{t} a_{0}(s) e^{\int_{s}^{t} a_{n-1}(\tau) d \tau} d s \\
& =e^{\int_{t_{0}}^{t} a_{n-1}(\tau) d \tau}\left[u_{0}-\varphi\left(t_{0}\right)\right]+\varphi(t) .
\end{aligned}
$$

If given the initial value $u_{0} \geq \varphi\left(t_{0}\right)$, then $u(t) \geq \varphi(t)>0$. 
Remark 4.10. Since the transformation of $u(t)=y^{1-n}(t)$, it requires $u(t)>0$, in addition,

$$
e^{\int_{t_{0}}^{t} a_{n-1}(\tau) d \tau}=e^{(n-1) \int_{t_{0}}^{t} a_{0}(\tau)\left(\int_{Y}^{\tau} a_{n}(\theta) d \theta\right)^{n-1} d \tau} \longrightarrow+\infty
$$

as $t \rightarrow+\infty$, that is to say, it requires $u_{0} \geq \varphi\left(t_{0}\right)$.

Under the transformation of $u(t)=y^{1-n}(t), y(t)=x(t)-\int_{\gamma}^{t} a_{n}(s) d s$, we can get the following.

If given the initial value

$$
x\left(t_{0}\right)=\left(u_{0}\right)^{-1 /(n-1)}+\int_{\gamma}^{t_{0}} a_{n}(s) d s<\varphi\left(t_{0}\right)^{-1 /(n-1)}+\int_{\gamma}^{t_{0}} a_{n}(s) d s \in \Omega_{1},
$$

then the unique solution $x(t)$ of (1.1) is given by

$$
x(t)=\left(u_{0} e^{\int_{t_{0}}^{t} a_{n-1}(\tau) d \tau}-(n-1) \int_{t_{0}}^{t} a_{0}(s) e^{t_{s}^{t} a_{n-1}(\tau) d \tau} d s\right)^{-1 /(n-1)}+\int_{\gamma}^{t} a_{n}(s) d s,
$$

and it is easy for us to know that $x(t) \in \Omega_{3}$.

If given the initial value

$$
x\left(t_{0}\right)=-\left(u_{0}\right)^{-1 /(n-1)}+\int_{\gamma}^{t_{0}} a_{n}(s) d s>-\left(\varphi\left(t_{0}\right)\right)^{-1 /(n-1)}+\int_{\gamma}^{t_{0}} a_{n}(s) d s \in \Omega_{2} ;
$$

then the unique solution $x(t)$ of (1.1) is given by

$$
x(t)=-\left(u_{0} e^{\int_{t_{0}}^{t} a_{n-1}(\tau) d \tau}-(n-1) \int_{t_{0}}^{t} a_{0}(s) e^{\int_{s}^{t} a_{n-1}(\tau) d \tau} d s\right)^{-1 /(n-1)}+\int_{\gamma}^{t} a_{n}(s) d s
$$

it is easy for us to know that $x(t) \in \Omega_{4}$.

Therefore, we can draw the following conclusion.

If given the initial value $x\left(t_{0}\right) \in \Omega_{1} \cup \Omega_{2}$, then $x(t) \in \Omega_{3} \cup \Omega_{4}$. 
Next, we prove that the periodic solution $\eta(t)$ of (1.1) is globally attractive in $\Omega_{3} \cup \Omega_{4}$ if given the initial value $x\left(t_{0}\right) \in \Omega_{1} \cup \Omega_{2}$.

$$
\begin{aligned}
|x(t)-\eta(t)| & =\left| \pm\left(u_{0} e^{\int_{t_{0}}^{t} a_{n-1}(\tau) d \tau}-(n-1) \int_{t_{0}}^{t} a_{0}(s) e^{\int_{s}^{t} a_{n-1}(\tau) d \tau} d s\right)^{-1 /(n-1)}\right| \\
& =\left(u_{0} e^{\int_{t_{0}}^{t} a_{n-1}(\tau) d \tau}-(n-1) \int_{t_{0}}^{t} a_{0}(s) e^{\int_{s}^{t} a_{n-1}(\tau) d \tau} d s\right)^{-1 /(n-1)} \\
& =\left(e^{\int_{t_{0}}^{t} a_{n-1}(\tau) d \tau}\left[u_{0}-\varphi\left(t_{0}\right)\right]+\varphi(t)\right)^{-1 /(n-1)} \\
& =\frac{\left|x\left(t_{0}\right)-\eta\left(t_{0}\right)\right|}{\left|x\left(t_{0}\right)-\eta\left(t_{0}\right)\right|^{n-1} \sqrt{e^{\int_{t_{0}}^{t} a_{n-1}(\tau) d \tau}\left[u_{0}-\varphi\left(t_{0}\right)\right]+\varphi(t)}} \\
& =\frac{\left|x\left(t_{0}\right)-\eta\left(t_{0}\right)\right|}{n-1 \sqrt{\left(1 / u_{0}\right)\left[e^{\int_{t_{0}}^{t} a_{n-1}(\tau) d \tau}\left[u_{0}-\varphi\left(t_{0}\right)\right]+\varphi(t)\right]}}
\end{aligned}
$$

by (4.53), it follows that

$$
\int_{t_{0}}^{t} a_{n-1}(s) d s \longrightarrow+\infty
$$

as $t \rightarrow+\infty$, from (4.63), we can get that the $\omega$-periodic solution $\eta(t)$ of (1.1) is globally attractive in $\Omega_{3} \cup \Omega_{4}$.

This is the end of the proof of Theorem 4.8 .

Theorem 4.11. Consider (1.1), $n$ is an even number, and $n \geq 2, a_{0}(t), a_{n}(t)$ are continuous $\omega$ periodic functions defined on $R$, if there is a constant $\gamma$ such that the following conditions hold:

$$
\begin{aligned}
& \left(H_{1}\right) a_{n-1}(t)=(1-n) a_{0}(t)\left(\int_{\gamma}^{t} a_{n}(s) d s\right)^{n-1} ; \\
& \left(H_{2}\right) a_{k}(t)=(-1)^{k} C_{n}^{k} a_{0}(t)\left(\int_{\gamma}^{t} a_{n}(s) d s\right)^{k}, k=1,2, \ldots, n-2 ; \\
& \left(H_{3}\right) m\left(a_{n}(t)\right)=0, a_{n}(t) \not \equiv 0 ; \\
& \left(H_{4}\right) a_{0}(t)<0, \int_{\gamma}^{t} a_{n}(s) d s \geq 0 ;
\end{aligned}
$$

then

(i) equation (1.1) has two w-periodic solutions $\eta(t), \phi(t)$, and they can be written as follows:

$$
\begin{gathered}
\eta(t)=\int_{\gamma}^{t} a_{n}(s) d s \\
\phi(t)=\left((n-1) \int_{t}^{+\infty} a_{0}(s) e^{\int_{s}^{t} a_{n-1}(\tau) d \tau} d s\right)^{-1 /(n-1)}+\int_{\gamma}^{t} a_{n}(s) d s
\end{gathered}
$$


(ii) if given any initial value $x\left(t_{0}\right) \in \Omega_{1}=\left\{x \mid x>\int_{\gamma}^{t_{0}} a_{n}(s) d s\right\}$, then the periodic solution $\eta(t)$ of (1.1) is globally attractive in $\Omega_{2}=\left\{x \mid x>\inf _{t \in[0, \omega]} \int_{\gamma}^{t} a_{n}(s) d s\right\}$.

Proof. The Proof of Theorem 4.11 is similar to that of Theorem 4.8, so we omit it here.

Theorem 4.12. Consider (1.1), $n$ is an even number, and $n \geq 2, a_{0}(t), a_{n}(t)$ are continuous $\omega$ periodic functions defined on $R$, if there is a constant $\gamma$ such that the following conditions hold:

$$
\begin{aligned}
& \left(H_{1}\right) a_{n-1}(t)=(1-n) a_{0}(t)\left(\int_{\gamma}^{t} a_{n}(s) d s\right)^{n-1} ; \\
& \left(H_{2}\right) a_{k}(t)=(-1)^{k} C_{n}^{k} a_{0}(t)\left(\int_{\gamma}^{t} a_{n}(s) d s\right)^{k}, k=1,2, \ldots, n-2 ; \\
& \left(H_{3}\right) m\left(a_{n}(t)\right)=0, a_{n}(t) \not \equiv 0 ; \\
& \left(H_{4}\right) a_{0}(t)>0, \int_{\gamma}^{t} a_{n}(s) d s \leq 0 ;
\end{aligned}
$$

then

(i) equation (1.1) has two w-periodic solutions $\eta(t), \phi(t)$, and they can be written as follows:

$$
\begin{gathered}
\eta(t)=\int_{\gamma}^{t} a_{n}(s) d s \\
\phi(t)=\left((n-1) \int_{t}^{+\infty} a_{0}(s) e^{\int_{s}^{t} a_{n-1}(\tau) d \tau} d s\right)^{-1 /(n-1)}+\int_{\gamma}^{t} a_{n}(s) d s
\end{gathered}
$$

(ii) if given any initial value $x\left(t_{0}\right) \in \Omega_{1}=\left\{x \mid x<\int_{\gamma}^{t_{0}} a_{n}(s) d s\right\}$, then the periodic solution $\eta(t)$ of (1.1) is globally attractive in $\Omega_{2}=\left\{x \mid x<\sup _{t \in[0, \omega]} \int_{\gamma}^{t} a_{n}(s) d s\right\}$.

Proof. The Proof of Theorem 4.12 is similar to that of Theorem 4.8, so we omit it here.

From Theorem 4.8, if $n=3$, it is easy for us to draw the following corollary.

Corollary 4.13. Consider Abel type differential equation (1.2), $a(t), d(t)$ are $\omega$-periodic continuous functions defined on $R$, if there is a constant $\gamma$ such that the following conditions hold:

$$
\begin{aligned}
& \left(H_{1}\right) b(t)=-3 a(t) \int_{\gamma}^{t} d(s) d s \\
& \left(H_{2}\right) c(t)=2 a(t)\left(\int_{\gamma}^{t} d(s) d s\right)^{2} \\
& \left(H_{3}\right) m(d(t))=0, d(t) \not \equiv 0 ; \\
& \left(H_{4}\right) a(t)>0 ;
\end{aligned}
$$

then 
(i) equation (1.2) has three w-periodic solutions $\eta(t), \phi(t), \chi(t)$, and they can be written as follows:

$$
\begin{gathered}
\eta(t)=\int_{\gamma}^{t} d(s) d s, \\
\phi(t)=\left(2 \int_{t}^{+\infty} a(s) e^{\int_{s}^{t} c(\tau) d \tau} d s\right)^{-1 / 2}+\int_{\gamma}^{t} d(s) d s, \\
x(t)=\left(2 \int_{t}^{+\infty} a(s) e^{\int_{s}^{t} c(\tau) d \tau} d s\right)^{-1 / 2}+\int_{\gamma}^{t} d(s) d s .
\end{gathered}
$$

(ii) If given any initial value $x\left(t_{0}\right) \in \Omega_{1} \cup \Omega_{2}$, then the periodic solution $\eta(t)$ of (1.2) is globally attractive in $\Omega_{3} \cup \Omega_{4}$, where

$$
\begin{aligned}
& \Omega_{1}=\left\{x \mid \int_{\gamma}^{t_{0}} d(s) d s \leq x<\left(\varphi\left(t_{0}\right)\right)^{-1 / 2}+\int_{\gamma}^{t_{0}} d(s) d s\right\}, \\
& \Omega_{2}=\left\{x \mid \int_{\gamma}^{t_{0}} d(s) d s \geq x>-\left(\varphi\left(t_{0}\right)\right)^{-1 / 2}+\int_{\gamma}^{t_{0}} d(s) d s\right\}, \\
& \Omega_{3}=\left\{x \mid \inf _{t \in[0, \omega]} \int_{\gamma}^{t} d(s) d s \leq x<\sup _{t \in[0, \omega]}\left((\varphi(t))^{-1 / 2}+\int_{\gamma}^{t} d(s) d s\right)\right\}, \\
& \Omega_{4}=\left\{x \mid \sup _{t \in[0, \omega]} \int_{\gamma}^{t} d(s) d s \geq x>\inf _{t \in[0, \omega]}\left(-(\varphi(t))^{-1 / 2}+\int_{\gamma}^{t} d(s) d s\right)\right\}, \\
& \varphi(t)=2 \int_{t}^{+\infty} a(s) e^{\int_{s}^{t} c(\tau) d \tau} d s .
\end{aligned}
$$

From Theorem 4.11 and 4.6, if $n=2$, it is easy for us to draw the following corollaries.

Corollary 4.14. Consider Riccati's equation (3.10), $a(t), c(t)$ are w-periodic continuous functions defined on $R$, if there is a constant $\gamma$ such that the following conditions hold:

$$
\begin{aligned}
& \left(H_{1}\right) b(t)=-a(t) \int_{\gamma}^{t} c(s) d s ; \\
& \left(H_{2}\right) m(c(t))=0, c(t) \not \equiv 0 ; \\
& \left(H_{3}\right) a(t)<0, \int_{\gamma}^{t} c(s) d s \geq 0 ;
\end{aligned}
$$

then

(i) equation (3.10) has two w-periodic solutions $\eta(t), \phi(t)$, and they can be written as follows:

$$
\begin{gathered}
\eta(t)=\int_{\gamma}^{t} c(s) d s, \\
\phi(t)=\left(\int_{t}^{+\infty} a(s) e^{\int_{s}^{t} b(\tau) d \tau} d s\right)^{-1}+\int_{\gamma}^{t} c(s) d s .
\end{gathered}
$$


(ii) If given the initial value $x\left(t_{0}\right) \in \Omega_{1}=\left\{x \mid x>\int_{\gamma}^{t_{0}} c(s) d s\right\}$, then the periodic solution $\eta(t)$ of (3.10) is globally attractive in $\Omega_{2}=\left\{x \mid x>\inf _{t \in[0, \omega]} \int_{\gamma}^{t} c(s) d s\right\}$.

Corollary 4.15. Consider Riccati's equation (3.10), $a(t), c(t)$ are w-periodic continuous functions defined on $R$, if there is a constant $\gamma$ such that the following conditions hold:

$$
\begin{aligned}
& \left(H_{1}\right) b(t)=-a(t) \int_{\gamma}^{t} c(s) d s ; \\
& \left(H_{2}\right) m(c(t))=0, c(t) \not \equiv 0 ; \\
& \left(H_{3}\right) a(t)>0, \int_{\gamma}^{t} c(s) d s \leq 0 ;
\end{aligned}
$$

then

(i) equation (3.10) has two w-periodic solutions $\eta(t), \phi(t)$, and they can be written as follows:

$$
\begin{gathered}
\eta(t)=\int_{\gamma}^{t} c(s) d s \\
\phi(t)=\left(\int_{t}^{+\infty} a(s) e^{\int_{s}^{t} b(\tau) d \tau} d s\right)^{-1}+\int_{\gamma}^{t} c(s) d s .
\end{gathered}
$$

(ii) If given any initial value $x\left(t_{0}\right) \in \Omega_{1}=\left\{x \mid x<\int_{\gamma}^{t_{0}} c(s) d s\right\}$, then the periodic solution $\eta(t)$ of (3.10) is globally attractive in $\Omega_{2}=\left\{x \mid x<\sup _{t \in[0, \omega]} \int_{\gamma}^{t} c(s) d s\right\}$.

\section{Concluding Remarks}

Generally, just as Ricatti's equation, (1.1) is nonintegrable, however in the present paper, we have got a sufficient condition which guarantees the integrability and have presented some exact periodic solutions of nonlinear equation if the coefficients of (1.1) satisfy certain conditions; moreover, we get that the periodic solutions are globally attractive in certain conditions. These conclusions will have certain applications.

\section{Acknowledgments}

The authors are highly grateful to the reviewers for their constructive comments. This work is supported by the National Nature Science Foundation of China (no. 11171135) and Jiangsu Province Innovation Project of Graduate Education (2011, no. 1221190037).

\section{References}

[1] J. P. M. Lebrun, "On two coupled Abel-type differential equations arising in a magnetostatic problem," Il Nuovo Cimento A, vol. 103, no. 10, pp. 1369-1379, 1990.

[2] F. Borghero and A. Melis, "On Szebehely's problem for holonomic systems involving generalized potential functions," Celestial Mechanics E Dynamical Astronomy, vol. 49, no. 3, pp. 273-284, 1990.

[3] S. S.. Bayin, "Solutions of Einstein's field equations for static fluid spheres," Physical Review D, vol. 18, no. 8, pp. 2745-2751, 1978. 
[4] A. García, A. Macías, and E. W. Mielke, "Stewart-Lyth second-order approach as an Abel equation for reconstructing inflationary dynamics," Physics Letters A, vol. 229, no. 1, pp. 32-36, 1997.

[5] V. R. Gavrilov, V. D. Ivashchuk, and V. N. Melnikov, "Multidimensional integrable vacuum cosmology with two curvatures," Classical and Quantum Gravity, vol. 13, no. 11, pp. 3039-3056, 1996.

[6] G. Haager and M. Mars, "A self-similar inhomogeneous dust cosmology," Classical and Quantum Gravity, vol. 15, no. 6, pp. 1567-1580, 1998.

[7] M. K. Mak and T. Harko, "Full causal bulk-viscous cosmological models," Journal of Mathematical Physics, vol. 39, no. 10, pp. 5458-5476, 1998.

[8] M. K. Mak and T. Harko, "Exact causal viscous cosmologies," General Relativity and Gravitation, vol. 30, no. 8, pp. 1171-1186, 1998.

[9] M. K. Mak and T. Harko, "Addendum to 'Exact causal viscous cosmologies'," General Relativity and Gravitation, vol. 31, no. 2, pp. 273-274, 1999.

[10] Y. Matsuno, "Two-dimensional dynamical system associated with Abel's nonlinear differential equation," Journal of Mathematical Physics, vol. 33, no. 1, pp. 412-421, 1992.

[11] G. L. Strobel and J. L. Reid, "Nonlinear superposition rule for Abel's equation," Physics Letters A, vol. 91, no. 5, pp. 209-210, 1982.

[12] J. L. Reid and G. L. Strobel, "The nonlinear superposition theorem of Lie and Abel's differential equations," Lettere al Nuovo Cimento, vol. 38, no. 13, pp. 448-452, 1983.

[13] M. K. Mak, H. W. Chan, and T. Harko, "Solutions generating technique for Abel-type nonlinear ordinary differential equations," Computers $\mathcal{E}$ Mathematics with Applications, vol. 41, no. 10-11, pp. 1395-1401, 2001.

[14] M. K. Mak and T. Harko, "New method for generating general solution of Abel differential equation," Computers \& Mathematics with Applications, vol. 43, no. 1-2, pp. 91-94, 2002.

[15] A. A. Zheltukhin and M. Trzetrzelewski, "U(1)-invariant membranes: the geometric formulation, Abel, and pendulum differential equations," Journal of Mathematical Physics, vol. 51, Article ID 062303 , p. 12, 2010.

[16] C. Y. He, Almost Periodic Functions and Differential Equations, Higher education press, Beijing, China, 1992.

[17] C. R. Li and S. J. Lu, "The qualitative analysis of n-species periodic coefficient, nonlinear relation, preycompetition systems," Applied Mathematics, vol. 12, no. 2, pp. 147-156, 1997. 


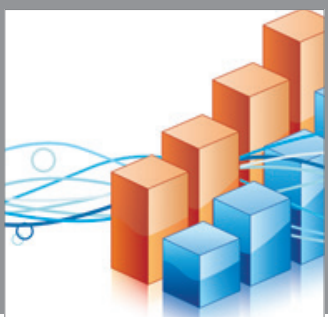

Advances in

Operations Research

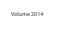

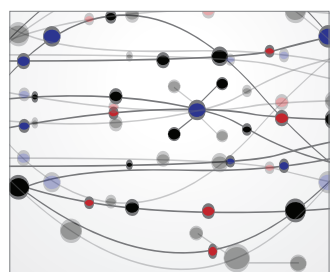

\section{The Scientific} World Journal
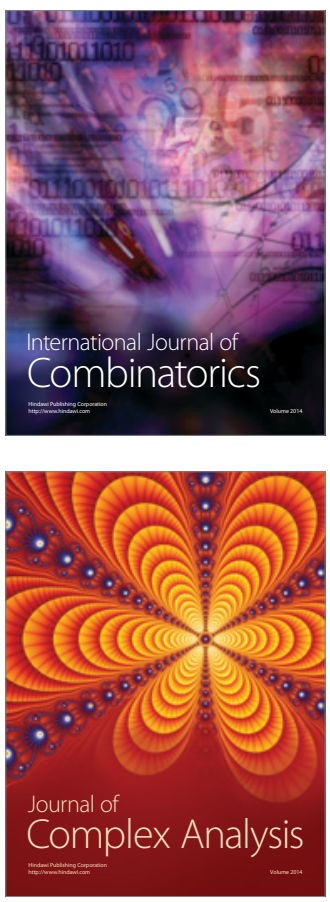

International Journal of

Mathematics and

Mathematical

Sciences
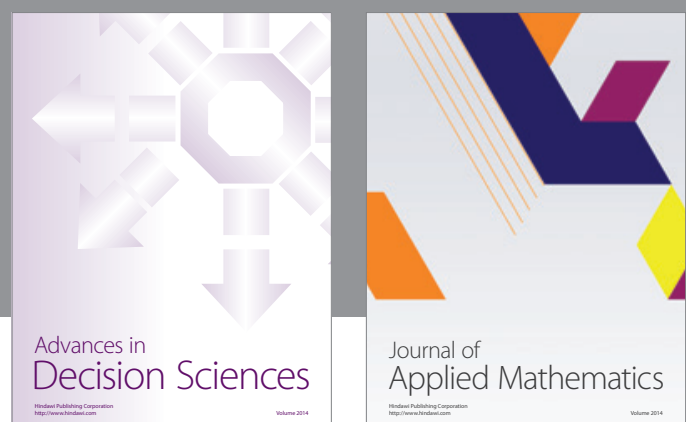

Journal of

Applied Mathematics
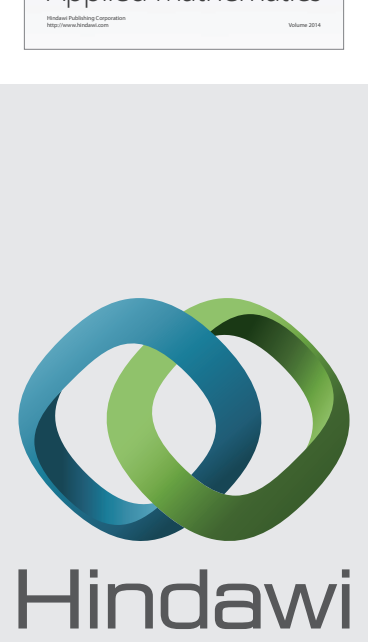

Submit your manuscripts at http://www.hindawi.com
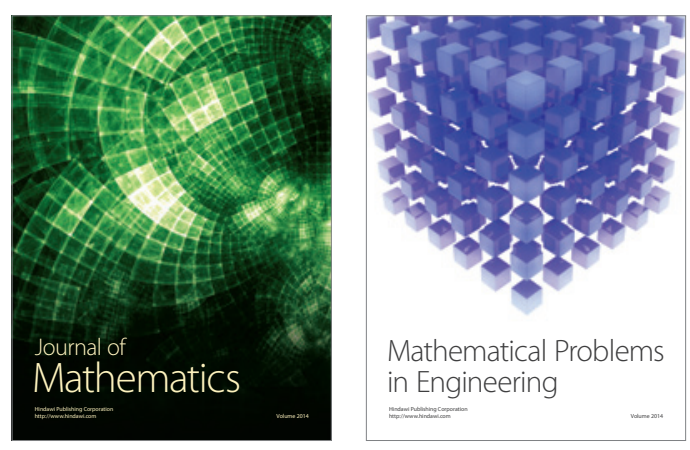

Mathematical Problems in Engineering
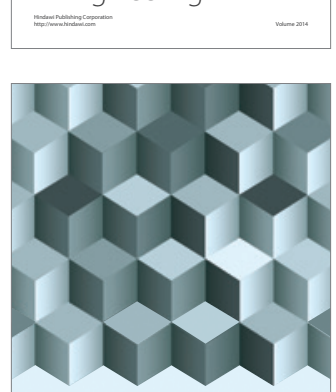

Journal of

Function Spaces
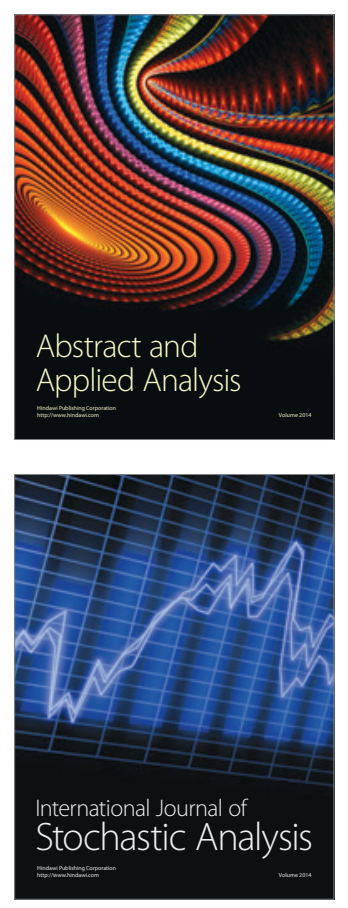

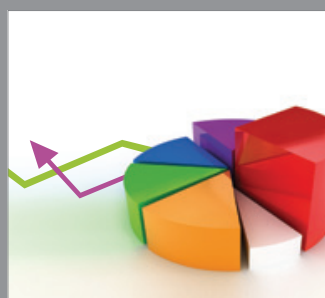

ournal of

Probability and Statistics

Promensencen
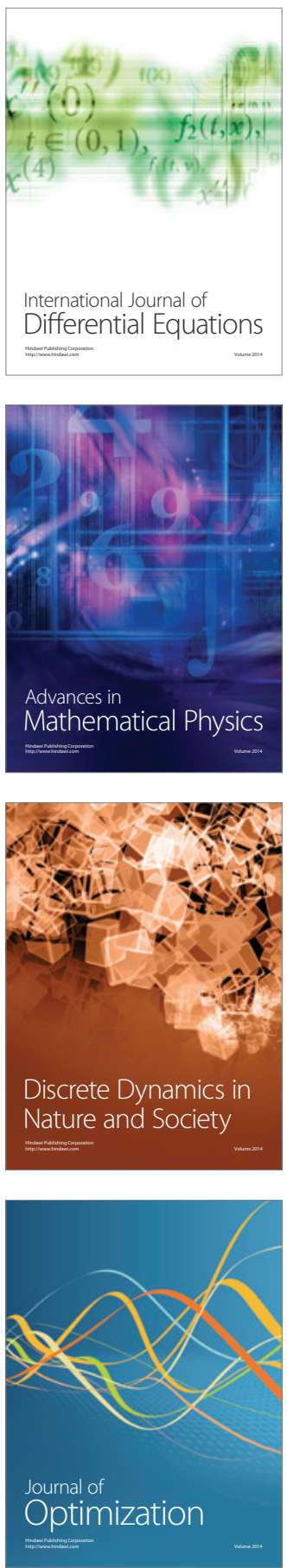